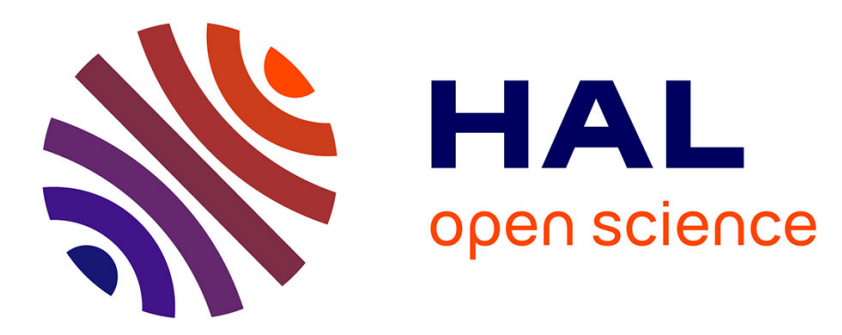

\title{
Qualitative Analysis of RSSI Behavior in Cooperative Wireless Body Area Networks for Mobility Detection and Navigation Applications
}

B Denis, Nicolas Amiot, Bernard Uguen, A Guizar, Claire Goursaud, A Ouni, C Chaudet

\section{To cite this version:}

B Denis, Nicolas Amiot, Bernard Uguen, A Guizar, Claire Goursaud, et al.. Qualitative Analysis of RSSI Behavior in Cooperative Wireless Body Area Networks for Mobility Detection and Navigation Applications. ICECS 2014, Dec 2014, Marseille, France. hal-01096509

\section{HAL Id: hal-01096509 https://hal.science/hal-01096509}

Submitted on 17 Dec 2014

HAL is a multi-disciplinary open access archive for the deposit and dissemination of scientific research documents, whether they are published or not. The documents may come from teaching and research institutions in France or abroad, or from public or private research centers.
L'archive ouverte pluridisciplinaire HAL, est destinée au dépôt et à la diffusion de documents scientifiques de niveau recherche, publiés ou non, émanant des établissements d'enseignement et de recherche français ou étrangers, des laboratoires publics ou privés. 


\title{
Qualitative Analysis of RSSI Behavior in Cooperative Wireless Body Area Networks for Mobility Detection and Navigation Applications
}

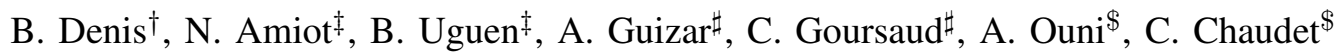 \\ ${ }^{\dagger}$ CEA-Leti Minatec, F-38054, Grenoble, France \\ ‡Université Rennes 1 - IETR, F-35042, Rennes, France \\ $\sharp$ INSA Lyon - CITI, F-69621, Lyon, France \\ $\$$ Telecom ParisTech - LINCS, F-75013, Paris, France \\ E-mails:benoit.denis@cea.fr
}

\begin{abstract}
In this paper, we account for radio-location experiments aiming at both indoor navigation and mobility detection applications for Wireless Body Area Networks (WBAN). This measurement campaign involved IEEE 802.15.4-compliant integrated radio devices organized within a full mesh topology over on-body and off-body links. The latter devices produce peerto-peer Received Signal Strength Indicators (RSSI) that could feed ranging, positioning or tracking algorithms. An in-depth behavioral analysis of the collected time-stamped radio-location metrics is thus proposed with respect to the captured human mobility (including body shadowing). Based on our observations and interpretations, practical insights are finally drawn in terms of system and algorithms design.
\end{abstract}

Index Terms-Body Shadowing, Cooperative Body Area Networks, Motion Capture, Navigation, Mobility Detection, Ranging, Received Signal Strength Indicator, Zigbee.

\section{INTRODUCTION}

Benefiting from recent advances in electronics and shortrange ultra-low power radio design, Wireless Body Area Networks (WBANs) have emerged as a new technology. It covers the needs of wide practical and innovative applications in security, healthcare, sports and entertainment. Recently, significant research interests have been devoted to locationaware functionalities, i.e. individual posture detection or indoor navigation. Most of the studies referenced in this field focus on WBAN measurements based on costly pieces of equipment, either for channel modeling purposes [1], [2] or for the validation of on-body localization algorithms based on impulse radio [3], [4]. Other approaches aim at characterizing mesh connectivity with integrated narrow-band devices from a pure communication-oriented perspective [5]. Finally some works simply rely on simulations [6]. But only rare contributions have considered using real devices to assess the actual performance of on-body nodes localization in large scale, while pointing out critical physical effects related to body mobility [7].

This paper accounts for an unprecedented and extensive localization-oriented measurement campaign based on integrated devices in a full mesh multi-standard WBAN context mixing both on-body and off-body links (i.e. at the body scale or with respect to fixed elements of infrastructure, respectively). During this campaign, a side infrared motion capture system was exploited for accurate space/time referencing [8]. Although both Impulse Radio - Ultra Wideband (IRUWB) [9], [10] and 2.45GHz IEEE 802.15.4-compliant [11] technologies have been considered under common deployment configurations, the paper focuses on the early exploitation of narrow-band data, as a preliminary step. The behavior of timestamped Received Signal Strength Indicators (RSSI) is thus illustrated and analyzed. In particular, we provide interpretations related to body shadowing under biomechanical constraints, conditionally informative (distance-dependent) path loss and relative body orientation with respect to fixed elements of infrastructure. These observations may have strong implications in terms of WBAN design and localization strategies for human mobility classification, detection or even tracking.

In Section II, we present the measurement set-up and scenarios. Then Section III provides illustrating examples of RSSI evaluations over representative links. In Section IV, we disclose insights for the design of location-enabled cooperative WBAN systems in terms of mobility and posture detection.

\section{Measurement Set-UP and Scenarios}

\section{A. Equipment}

a) IEEE 802.15.4-compliant radio devices: The involved HiKoB FOX wireless sensors [11] embed a processor and a radio chipset whose physical layer is IEEE 802.15.4-compliant in the $2.45 \mathrm{GHz}$ ISM band. The FOX radio chipset gives access to the average RSSI obtained over the last 8 received modulation symbols. Similarly to [5], a Time Division Multiple Access (TDMA) is considered, each node transmitting periodically in a pre-determined timeslot. An additional longer timeslot is dedicated for logging onto the $\mu$-SD card all the information locally collected by the nodes during each frame. The corresponding protocol adapts the frame duration depending on the number of nodes (e.g. 26ms for 16 nodes). During this new measurement campaign, up to 12 nodes have been deployed on the subject bodies, whereas 4 more nodes were placed in the immediate environment, serving as fixed 


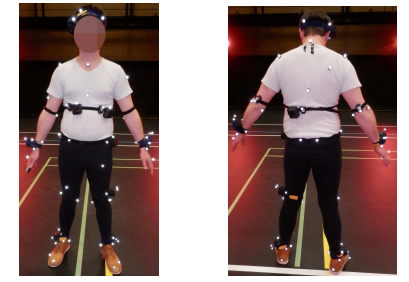

Fig. 1. Front and back pictures of the equipped subject, including 12 on-body HikoB nodes and tens of Vicon markers.

anchors of infrastructure, $A P i, i=1 \ldots 4$, at $1.06 \mathrm{~m}$ of height (Fig. 1 and 2).

b) Reference motion capture system: Besides radio devices, the Oxford Metrics Group's Vicon motion capture system, traditionally used to analyze elite athletes' movements [8], was involved in our measurement campaign. In particular, it was used to deliver high-precision spatial referencing (i.e. seen as ground truth), accurate synchronization and mobility modeling for the deployed on-body nodes. The Vicon capture was performed at $100 \mathrm{~Hz}$ using 12 infrared cameras surrounding the scene. In each experiment, we equipped the test subject with several tens of markers placed on the on-body devices and on anatomical landmarks to precisely reconstruct each body parts 3D position and orientation. The acquired information is used in the following to compute the exact distances separating the radio nodes and determine radio obstructions at any time.

\section{B. Experimental Scenario}

The experiments were realized in a gymnasium dedicated to motion capture studies at ENS/IRISA Cachan Bretagne, France. The capture zone was restricted to a $13 \mathrm{mx} 8 \mathrm{~m}$ area, including the 4 anchors and surrounded by the Vicon infrastructure (See Fig. 1 and 2). The considered canonical scenario deliberately mixed moderate pedestrian walking and static poses (for mobility and attitude detection), each trial lasting for about $110 \mathrm{sec}$. The subject was holding a smartphone in his right hand to mimic the use of a navigation display device.

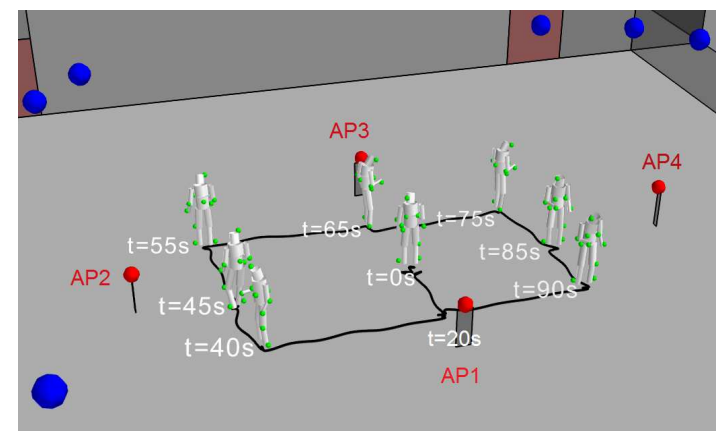

Fig. 2. Partial view of deployment scenario, including 4 fixed anchors (red spheres), 12 infrared cameras (big blue spheres), the followed trajectory (black ground path), and time-stamped snapshots of the body poses (articulated chains of light gray cylinders) with on-body radio nodes (green spheres).

At the beginning of the acquisition, after synchronizing motion capture and radio sub-systems through the crossdetection of a specific arm gesture, the subject body was standing right in the middle of the scene, facing each anchor for approximately $5 \mathrm{sec}$ each and rotating over the 4 anchors sequentially. Then at time $t=20 \mathrm{sec}$, the subject started moving along a rectangular trajectory centered on the starting point. The sequence is then divided into 9 moving subsequences, where the subject walks from one point of interest to another, with 9 intercalated static sequences. For the sake of illustration, Fig. 2 reports time-stamped snapshots of the body posture and orientation at different instants (corresponding to the 9 sequences) spanning from $t=0 \mathrm{sec}$ till $t=90 \mathrm{sec}$.

\section{ILlustration AND ANALYsis of RSSI BehaVIOR}

In this section, we qualitatively interpret the evolution of RSSI over on-body and off-body links separately, based on the high-definition body motion capture and scenario time-line.

\section{A. On-Body RSSI Behavior}

This study consists in analyzing the behavior of on-body links related to the nodes under mobility. Time-dependent RSSI measurements are evaluated over different links associated with the highly mobile node placed on the Right Wrist (RW). The links exhibiting similar behaviors over time have been grouped into 4 subsets: Subset 2 gathering the links between WR and the right upper part of the body, mostly in radio visibility (i.e. HeadRight, TorsoRight, ElbowRight, HipRight); Subset 3 gathering the links between RW and the left upper part of the body (plus the back), most often and strongly shadowed (i.e. Tor soTopLeft, BackCenter, ElbowLeft) and Subset 4 gathering links between RW and other fast moving terminations (i.e. WristLeft, KneeLeft, AnkleLeft, AnkleRight). One more Subset 5, gathering the links between Left Wrist (LW) and nodes placed at HeadRight, TorsoTopLeft, BackCenter and ElbowLeft, is added for comparison with RW. Our Subsets labelling arbitrarily respects that of the acquisition for processing convenience (unshown Subset 1 stands for the links wrt. anchors).

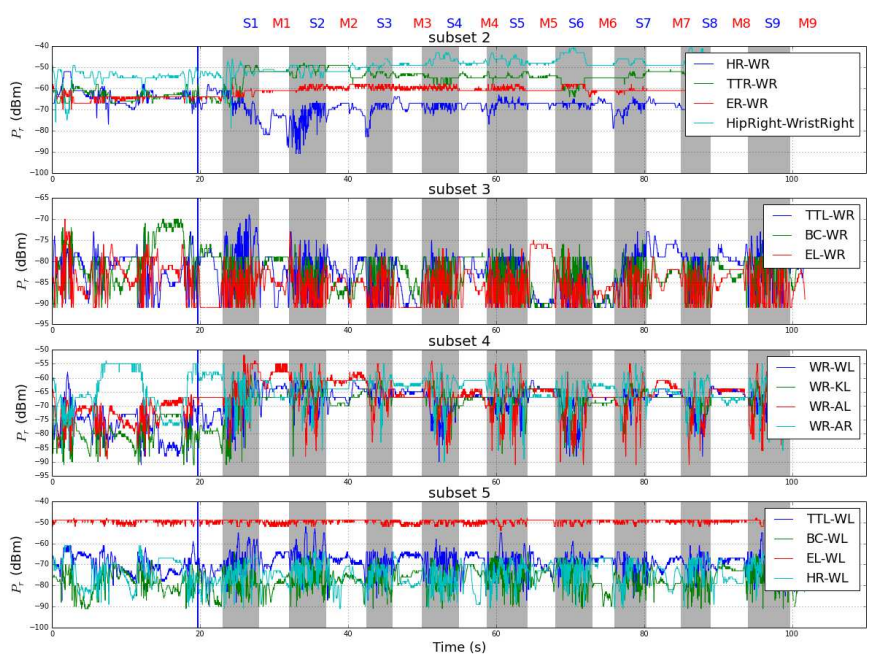

Fig. 3. RSSI evolution during the 9 sequences (white regions for static positions and gray regions for walking) over different classes of links: (Subset 2) RW - nodes placed on the right upper part of the body; (Subset 3) RW nodes placed on the left upper part of the body; (Subset 4) RW - alternative peripheral nodes; (Subset 5) LW - mixed nodes. 
First of all, the 9 sequences described in Section II-B are clearly noticeable on all the curves of Fig. 3, even if they are more visible in Subsets 3 and 4. Periods of fast signal variations (resp. slow signal variations) represent the portions of time when the subject is walking (resp. static). Moreover, the results of Subset 2 show a quasi-constant RSSI value with low dispersion and slow variations compared to the results of Subset 5 for the left wrist. In fact, the right hand holding a smartphone is time-invariant and in Line of Sight (LoS) with respect to the body torso and the head, whereas the left hand is mobile and in Non Line of Sight (NLoS). Other locally periodic effects over walking portions of time, caused by cyclic movements and fast accelerations of other peripheral nodes over repeated gaits, are more directly revealed by Subset 4 results, as expected. Subset 3 clearly illustrates the effect of body shadowing, leading to higher RSSI versatility and dispersion, but also stronger average attenuations. Finally, we also observe in Subset 3 a much higher packet loss rate (i.e. the received power being below the Rx RSSI sensitivity of $-90 \mathrm{dBm})$. In this case on- and/or off-body cooperation would be beneficial to communication QoS.

\section{B. Off-Body RSSI Behavior}

This study focuses on the channel characteristics of offbody links. We are particularly interested by the links between access points and the TorsoTopLeft (TTL) and BackCenter (BC) nodes, since the latter experience low relative on-body mobility while the subject is walking and they offer orthogonal obstruction conditions. The RSSI evolution over these links and their corresponding inverse squared distances in log-scale are shown on Fig. 4 for the 40 first sec of the acquisition. For the two links with respect to $A P 1$, the body obstruction status is superposed on the evolution of the ground truth quantity for illustration purposes. This status has been retrieved based on motion capture acquisition after simplifying the body as a set of 11 cylinders, thus determining intersections of the LoS path by one of these cylinders at any time.
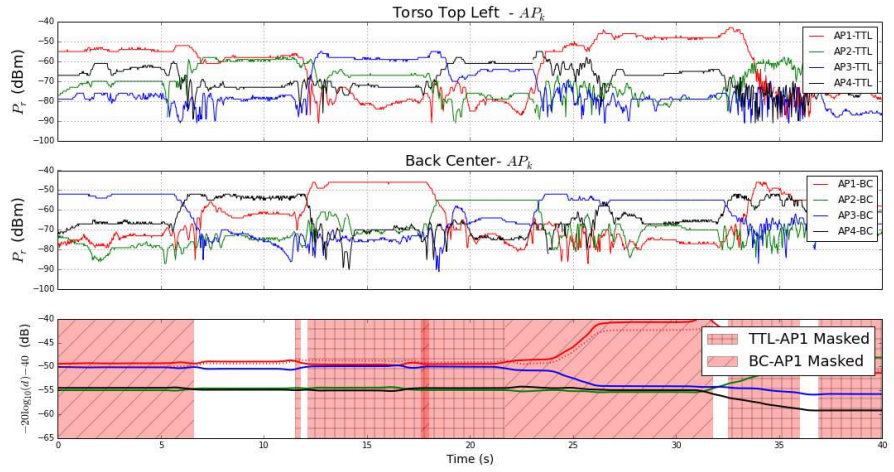

Fig. 4. Comparison between measured RSSI over $T T L-A P_{i} / B C-$ $A P_{i}$ links and their corresponding distance-dependent path loss, where NLoS conditions between $T T L / B C$ and $A P 1$ are represented by crosshatch (Zoom on the first $40 \mathrm{sec}$ only).

During the first $20 \mathrm{sec}$ of the sequence, the subject is facing sequentially the anchors $\{A P i\}_{i=1 \ldots 4}$. Accordingly, the distances with respect to $A P 1$ and $A P 3$ (resp. to $A P 2$ and
$A P 4)$ are constant and approximately equal. The distances between the subject and $A P 2$ and $A P 4$ being larger than for $A P 1$ and $A P 3$ according to the scene configuration, the log inverse of their squared distances naturally appears to be smaller. The observed RSSI variations (and relative mistmatch to the model) are thus not due to the variation of distance but essentially to the body shadowing status. For instance, when the subject is facing $A P 1$, a deep body shadowing is produced over the link $A P 2-T T L$, leading to the low observed level of $-70 \mathrm{dBm}$. For the second portion of time, when the subject is now facing $A P 2$ and the link status switches rapidly to LoS conditions, the RSSI level increases suddenly up to -60 $\mathrm{dB}$. For the third static posture, the subject is facing AP3 but the device placed on the left part of torso remains in optical visibility from $A P 2$, and the RSSI level drops down to $-66 \mathrm{~dB}$. The fourth static posture corresponding to the subject facing $A P 4, A P 2$ is strongly obstructed and RSSI is dropping at an even lower level of $-80 \mathrm{dBm}$. When the subject is walking in visibility in the direction of $A P 1$, the RSSI increases rapidly in accordance with the inverse square of the actual distance. Overall, the full pattern of the 4 observed RSSI values with respect to the anchors (especially the differential behaviours observed at $T T L$ and $B C$ on-body nodes) seems to provide relevant indications on both the anchors proximity and the relative body orientation. This result is valid not only during the stance phases but under mobility as well.

\section{IMPLICATIONS FOR RSSI-BASED MOBILITY DETECTION AND NAVIGATION IN COOPERATIVE WBANS}

\section{A. Blind Classification and Detection of Mobility Patterns}

As seen in Fig. 3, the RSSI evolution jointly observed over different groups of on-body links is clearly an indicator of the mobility status. Accordingly, it could be beneficial to human activity and body posture detection. For instance, patterns of empirical statistics computed on-line over judiciously chosen Subsets of on-body links (e.g. calculating the sample mean, standard deviation and kurtosis) of the RSSI (or even the instantaneous packet loss rate) measured over time could be used for preliminary learning and subsequent decision (e.g. based on machine learning or Bayesian tools) within a finite alphabet of possible postures (e.g. static vs. walking, right arm ahead or naturally balancing, etc.). As an example, Fig. 5 represents uniquely the RSSI standard deviation for the same links Subsets as that of Fig. 3 and for different mobility regimes. We notice that high (resp. low) relative deviations generally account for mobile (resp. static) sequences, whatever the link Subset, but even more remarquably for the links involving peripheral nodes like in Subsets 2 and 4. Accordingly, implementing joint Bayesian decision rules relying on several combinations of links and/or Subsets seem feasible to reinforce mobility detection rates, while preventing from false alarms. One could also make easily the distinction between different arm postures, based on the absolute deviation levels observed for Subsets 2 (i.e. low in average and sensitive to mobility) and 5 (higher in average and almost insensitive to mobility), i.e. relying on links that involve the two wrists (except the links to 
the elbows, which obviously exhibit the same low deviations regardless of the posture).
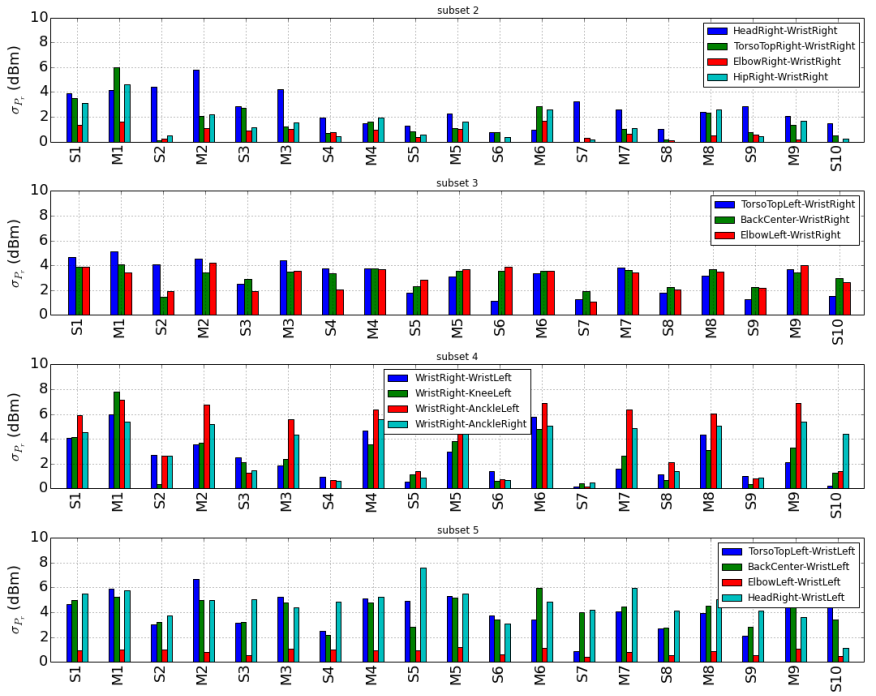

Fig. 5. RSSI standard deviation for distinct links and mobility sequences.

\section{B. Improved Personal Indoor Navigation}

Under the conventional log-normal shadowing modeling assumption, the best achievable RSSI-based ranging precision is proportional to a ratio binding the shadowing standard deviation and the path loss exponent (e.g, [12]). Intuitively, a high ratio implies that the deterministic power loss as a function of the distance is no longer significant nor dominating in comparison with the shadowing dispersion, thus making the interpretation of any RSSI readings hazardous from a ranging perspective. As discussed in [13], path loss exponents are very low over off-body links in frank NLoS cases (typically $<1$ ), whereas the measured power dispersion is adversely large (by several $\mathrm{dBm}$ ). But body obstructions cannot be classified so easily into binary cases (i.e. visibility or not) over these offbody links and there is a continuum of possible shadowing configurations, as a function of the relative subject orientation, depending whether the body partially or totally obstructs the propagation of direct radio waves (e.g. leading to power fluctuations of $25 \mathrm{~dB}$ over a body rotation of $360^{\circ}$ [2]).

Thus, instead of considering the conventional representation, conditional distance-dependent path loss models could be derived (i.e. conditioned on prior NLoS detection), whose parameters depend on the body shadowing status (e.g. including a systematic and constant additional loss) and even possibly, on the body relative orientation with respect to the LoS path (i.e. with a deterministic and monotonic angular-dependent additional loss). Based on our experiments, the extraction of such model parameters would be possible, based on the motion capture information and body-cylinder approximation at any time (though not shown here for paper length constraints). In a future study, the residuals obtained after LS model fitting to experimental RSSI data will be compared for the different modeling assumptions mentioned above in order to highlight the impact of prior NLoS detection on off-body ranging quality and thus, on navigation. Finally, Fig. 4 indicates that the judicious placement of two on-body nodes only, respectively on the torso and back, is definitely relevant into navigation scenarios, leading to orthogonal obstruction conditions and spatial diversity with respect to the infrastructure under mobility.

\section{CONCLUSION}

This paper experimentally analyzed the RSSI behavior in full mesh WBANs under a mixing on-body and off-body cooperative scenario. Our results showed qualitative correlations between the RSSI evolution and the ground truth inter-node distances, but also the difficulty of unconditionally exploiting such power measurements as explicit (i.e. in absolute $\mathrm{dBm}$ scale) range-dependent radio metrics for further higher level localization algorithms in lack of additional information about the body shadowing status. Further studies shall investigate contextual body shadowing mitigation for RSSI-based ranging and navigation over off-body links, as well as mobility/posture detection algorithms based on on-body links. A rather similar analysis shall be also conducted on IR-UWB temporal radiolocation metrics such as Round Trip - Time of Flight (RT-ToF), still based on the same measurement campaign.

\section{ACKNOWLEDGMENT}

This work has been carried out in the frame of the CORMORAN project, which is funded by the French National Research Agency (ANR) under the contract number ANR11-INFR-010. We thank all the involved researchers from ENS/IRISA and M2S Lab for their precious support during the measurement campaign.

\section{REFERENCES}

[1] S.L. Cotton, R. D'Errico and C. Oestges, "A review of radio channel models for body centric communications," in Radio Science, vol.49, is.6, pp. 371388, June 2014

[2] R. Rosini and R. D'Errico, "Off-body Channel Modelling at $2.45 \mathrm{GHz}$ for Two Different Antennas," Proc. EUCAP'12, pp. 3378-3382, 2012

[3] Z. Mekonnen, E. Slottke, H. Luecken, C. Steiner, and A. Wittneben, "Constrained Maximum Likelihood Positioning for UWB based Human Motion Tracking,' Proc. IPIN'10, 2010

[4] H. Shaban, M. El-Nasr, and R. Buehrer, "Toward a Highly Accurate Ambulatory System for Clinical Gait Analysis via UWB Radios," in IEEE Trans. Information Technology in Biomedicine, 14(2):284-291, 2010

[5] M. Lauzier, P. Ferrand, A. Fraboulet, H. Parvery, J.-M. Gorce, "Full Mesh Channel Measurements on Body Area Networks under Walking Scenarios," Proc. IEEE EuCAP'13, pp.3508,3512, April 2013

[6] J. Hamie, B. Denis, and C. Richard, "Joint Motion Capture and Navigation in Heterogeneous Body Area Networks with Distance Estimation over Neighborhood Graph," Proc. WPNC'13, Dresden, March 2013

[7] J. Hamie, Mickael Maman, and B. Denis, "On-Body Localization Experiments using Real IR-UWB Devices” In ICUWB'14, Paris, Sept. 2014

[8] B. Bideau, et al. "Using Virtual Reality to Analyze Sports Performance," in IEEE Computer Graphics and Applications, vol.30, no.2, pp.14-21, March-April 2010.

[9] M. Pezzin, D. Lachartre, "A Low Power, Low Data Rate Impulse Radio Ultra Wide Band Transceiver," Proc. FUNEMS'10, June 2010

[10] "BeSpoon SpoonPhone and tags," http://spoonphone.com/en/, 2014.

[11] "HiKoB FOX sensor," http://www.hikob.com/hikob-fox, 2012.

[12] Y. Qi and H. Kobayashi, On Relation among Time Delay and Signal Strength based Geolocation Methods, in Proc. IEEE GLOBECOM03, vol. 7, pp. 40794083, San Francisco, Dec. 2003.

[13] J. Hamie, "Contributions to Cooperative Localization Techniques within Mobile Wireless Body Area Networks,' PhD Manuscript, University of Nice-Sophia Antipolis, Nov. 2013 